\title{
Examining the Effects of Fluid Velocity Gradients on 4D Digital Holographic PIV/PTV Measurements
}

\author{
Y. J. Xia*, B. Sun, G. A. Ahmed, J. Soria \\ ${ }^{1}$ Laboratory for Turbulence Research in Aerospace \& Combustion (LTRAC), \\ Department of Mechanical and Aerospace Engineering, Monash University (Clayton \\ Campus), Melbourne, Victoria 3800, Australia \\ *Yuan.Xia@monash.edu
}

\begin{abstract}
4D digital holographic PIV/PTV (4D-DHPIV/PTV) methods have demonstrated theoretical viability due to their relative ease of setup and high spatial resolution (Soria (2018)). This study investigates how velocity gradients related to different flow regimes and their magnitudes affect 3-component-3-dimensional (3C-3D) digital holographic PIV measurement uncertainty.
\end{abstract}

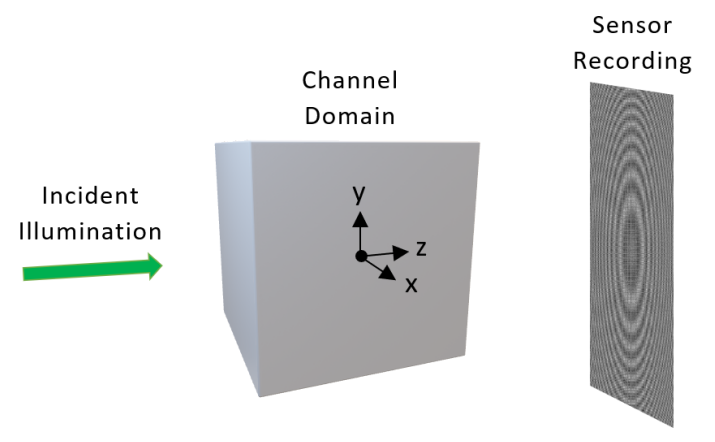

Figure 1: Diagram of simulated digital holographic PIV/PTV setup

The error introduced by velocity gradients within the interrogation volume is studied by simulating particles in a velocity field, with a given constant velocity gradient superimposed on a uniform flow from which a time-series of hologram pairs are generated and the 3C-3D velocity fields and their errors are determined using 4D-DHPIV/PTV Sun et al. (2020). Hologram pairs are simulated by modelling the propagation and particlediffraction of coherent laser light using the angular spectrum method (Goodman (1996)). The hologram reconstruction then involves direct reconstruction, followed by deconvolution, a particle position refinement and a hologram subtraction step (Sun et al. (2020)). The particle positions obtained from 4D-DHPIV/PTV are then used to resolve particle displacement measurements using 3D cross-correlation digital analysis with a 3D Gaussian fit to sub-pixel resolution (Soria (2006)).

The effects of velocity gradients on the displacement uncertainty and bias error have been investigated by undertaking Monte Carlo simulations under a range of velocity gradient environments. Specifically, 5 common velocity gradients have been studied, which included pure strain, pure vorticity and $x, y$ and $z$-directional shear. 


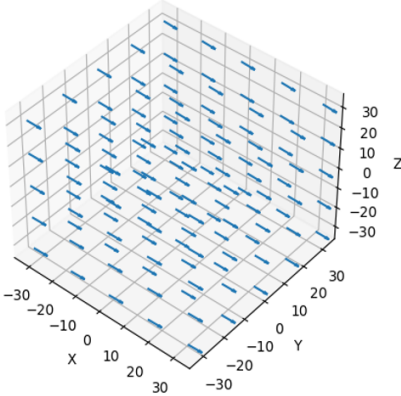

(a) Uniform mean velocity

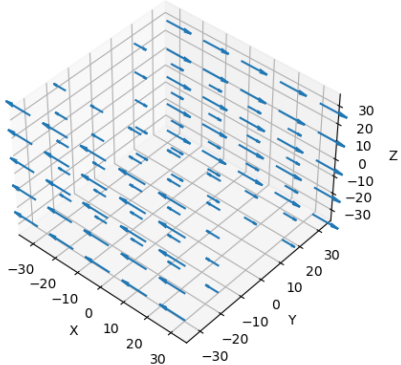

(d) Pure x shear velocity

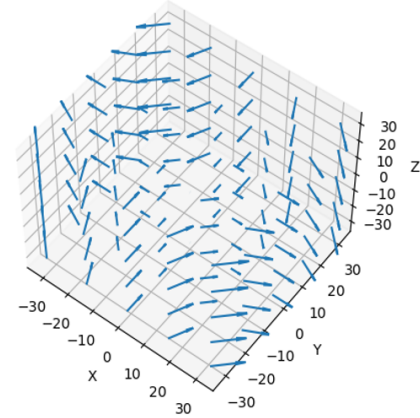

(b) Pure strain velocity

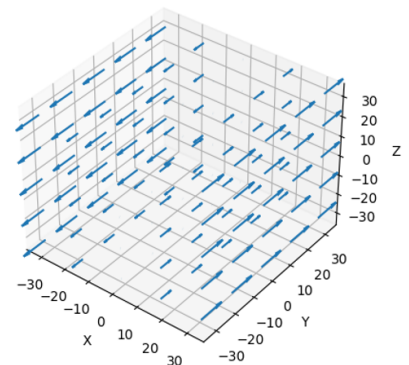

(e) Pure y shear velocity

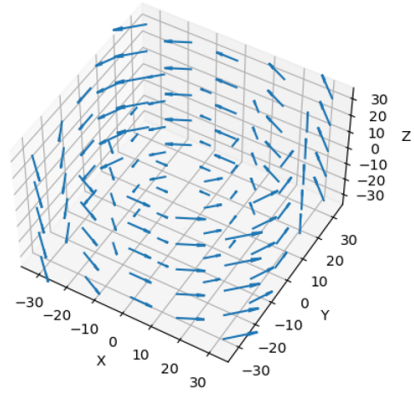

(c) Pure vorticity velocity

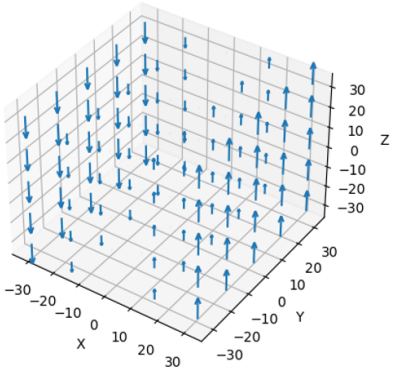

(f) Pure z shear velocity

Figure 2: The 6 different flow regime displacement vector plots used in this study. All test cases are subjected to a mean velocity field (a) superimposed with a velocity gradient flow regime (b-f). Vector plots ( $b-f)$ have been scaled by a factor of 2 for visualisation purposes. All axes are in pixels.

The results indicate that the novel 4D-DHPIV/PTV has poorer accuracy and precision in the z-propagation axis, resulting in larger minimum uncertainties and bias errors. The errors in the $z$ axis are also significantly less affected by velocity gradients in the $z$ direction when compared to the effects of $\mathrm{x}$ and $\mathrm{y}$ directional velocity gradients on $\mathrm{x}$ and $\mathrm{y}$ errors respectively. Furthermore, the rate of cross-correlation maximum and SNR decrease are approximately 1.36 times slower due to velocity gradients in the $\mathrm{z}$ axis than other axes.

\section{Acknowledgements}

The authors gratefully acknowledge the support of this project through ARC and NCMAS (NCI, Massive). Yuan Jing Xia gratefully acknowledges the support through James McNeill Foundation and Monash University Scholarships.

\section{References}

Goodman J (1996) Foundations of scalar diffraction theory. in Introduction to Fourier Optics. pages 55-57. McGraw Hill, New York City, USA

Soria J (2006) Particle image velocimetry - application to turbulence studies. in Lecture Notes on Turbulence and Coherent Structures in Fluids, Plasmas and Nonlinear Media. pages 307-347. World Scientific, Singapore

Soria J (2018) Three-component three-dimensional (3c-3d) fluid flow velocimetry for flow turbulence investigations. in Proceedings of the 21st Australasian Fluid Mechanics Conference, Adelaide, Australia, December 10-13

Sun B, Ahmed A, Atkinson C, and Soria J (2020) A novel 4d digital holographic piv/ptv (4d$\mathrm{dhpiv} / \mathrm{ptv}$ ) methodology using iterative predictive inverse reconstruction. Measurement Science and Technology 31 